\title{
PCSK9 Inhibitors and Neurocognitive Adverse Events: Exploring the FDA Directive and a Proposal for $N$-of-1 Trials
}

\author{
Kristopher J. Swiger $^{1} \cdot$ Seth S. Martin ${ }^{1}$
}

Published online: 20 May 2015

(c) Springer International Publishing Switzerland 2015

\begin{abstract}
Proprotein convertase subtilisin-kexin type 9 (PCSK9) inhibitors are a novel class of medications that greatly lower low-density lipoprotein cholesterol (LDL-C) by upregulating LDL receptor availability. In early 2014, the US Food and Drug Administration (FDA) directed developers of PCSK9 inhibitors to monitor neurocognitive adverse effects and consider neurocognitive testing in at least a subset of participants in ongoing late-stage trials. Available trial evidence indicates that neurocognitive adverse events may occur more commonly in individuals receiving an antibody to PCSK9, but these events are uncommon and have not been associated with on-treatment LDL-C levels. Moreover, it is unclear to what extent closer monitoring of trial participants allocated to PCSK9 inhibitors has led to an ascertainment bias. Regardless, further trial data are needed, and long-term outcomes trials are ongoing, with at least one including a neurocognitive substudy. Considering lessons learned from the statin experience, high-quality prospective cohort studies and randomized trials may not be enough to allay concerns or settle debate since the focus of effect in these studies is the group average. Therefore, we suggest that $n$-of- 1 trials could be considered to bring the focus to the individual while retaining the benefits of blinding and randomization in evidence generation. Ultimately, any neurocognitive adverse effects that might exist with PCSK9 inhibition and
\end{abstract}

Seth S. Martin

smart100@jhmi.edu

Kristopher J. Swiger

kswiger2@jhmi.edu

1 The Johns Hopkins Ciccarone Center for the Prevention of Heart Disease, 600 N. Wolfe Street Blalock 524-C, Baltimore, MD 21287, USA lipid lowering must be weighed against potential benefits of therapy, including avoidance of myocardial infarction and stroke, and a reduced risk of dementia due to neurovascular benefits from long-term lipid lowering.

\section{Key Points}

Available trial evidence indicates neurocognitive adverse events are uncommon but may occur more frequently in patients allocated to the proprotein convertase subtilisin-kexin type 9 (PCSK9) treatment group.

$\mathrm{N}$-of- 1 trials may be a potential solution for patients experiencing neurocognitive adverse events while taking PCSK9 inhibitors.

Providers should inform patients of the potential cardiovascular and neuroprotective benefits of PCSK9 therapy as well as the potential risk for neurocognitive adverse effects.

\section{Introduction}

Proprotein convertase subtilisin-kexin type 9 (PCSK9) inhibitors, which are injectable antibodies to PCSK9, are generating excitement for their ability to greatly lower lowdensity lipoprotein cholesterol (LDL-C) by upregulating low-density lipoprotein receptor (LDLR) availability. Later in 2015, it is expected that the US Food and Drug Administration (FDA) will decide if and for whom regulatory approval will be granted on the grounds of efficacy and 
safety records from existing trial data. It is conceivable that approval could be issued for patients with familial hypercholesterolemia, statin intolerance, or even broader cardiovascular indications. Additionally, pivotal clinical trials powered for atherosclerotic cardiovascular disease outcomes are ongoing in patients with established cardiovascular disease or cardiovascular risk factors who are already on background lipid therapy. In early 2014, reacting to early safety signals with the novel drug class, the FDA issued a directive to developers of PCSK9 inhibitors to assess the potential for neurocognitive adverse effects [1]. Along with monitoring for adverse neurocognitive events, the FDA requested that developers determine the feasibility of including neurocognitive testing in at least a subset of participants in ongoing late-stage trials.

Concerns about cognition with lipid lowering are not new. Memory complaints may be, at least according to one targeted survey, the second most common patient-reported adverse effect for statin medications [2]. In 2012, the FDA mandated a black box warning change for all statin drugs citing "ill-defined memory loss or impairment" [3]. Despite systematic reviews and clinical practice guidelines [4] finding that high-quality evidence does not support a claim of cognitive harm, the label change persists. Adding to confusion, statin therapy is associated with a decreased incidence of dementia in high-quality prospective cohort studies [5]. There may be lessons from the statin experience that will help sort through PCSK9 safety concerns.

In this context, we discuss the possibility of neurocognitive impairment with any lipid-lowering therapy and how to possibly reconcile discordant reports. Psychiatric effects, such as affective disorders or mood changes, are not the intended focus of this review. Next, we discuss whether concern for adverse neurocognitive effects extends to PCSK9 inhibitors. Then, we examine evidence to date from PCSK9 trials, take a look into the ongoing studies that are planned, and explore how $n$-of- 1 trials might be a useful tool for rigorous patient-centered safety evaluation.

\section{Why It is Biologically Plausible that Lipid-Lowering Therapy Could Cause Cognitive Adverse Effects}

While statins have received most of the attention for potential neurocognitive effects through lipid lowering, such a mechanism could in theory apply to any single lipid-lowering intervention. The effect would be theoretically expected to relate to the magnitude of lipid lowering, with a threshold below which harm could occur. Hence, the true underlying source of cognitive decline, if such an effect were to be observed, would be the impact of lowering lipid levels on the functions of the central nervous system (CNS).
The brain houses an estimated $25 \%$ of the body's total cholesterol [6]. Cholesterol is a principal component of myelin, a fatty sheath that serves an essential role in cellular signaling and blood-brain barrier integrity [7]. Statins, regardless of their lipophilicity and ability to penetrate the blood-brain barrier, alter the composition of brain lipids in rat models [8]. Additionally, reduced serum cholesterol may also enhance blood-barrier permeability, exposing the CNS to normally barred toxins [9]. It is therefore at least biologically plausible that lowering serum cholesterol could impact brain function, particularly in cell signaling.

PCSK9 inhibitors dramatically lower LDL-C by as much as $75 \%$ according to phase III data [10-12]. But should this impact on cholesterol homeostasis raise legitimate concerns about the effect of lipid-lowering therapy and cognitive problems? While the animal studies referenced above suggest an influence of serum on CNS cholesterol, the real question is whether this influence reaches a threshold to cause functional impairment. To our knowledge, no studies - animal or human-have demonstrated a causal link between CNS cholesterol reductions (initiated by lipid-lowering therapy) and functional impairment. Additionally, the average LDL-C level in Western society (115 mg/dL in 2005-2006 in the USA [13]) far exceeds the approximately $50 \mathrm{mg} / \mathrm{dL}$ seen in hunter-gatherers, human neonates, and genetically similar primates [14]. It seems unlikely that a reduction from our population's "normal" serum cholesterol values to the physiological (and nearly arteriosclerosis-free) range seen in our direct ancestors would result in alterations to brain function. Furthermore, persons born with genetic loss-of-function variants in PCSK9 have not shown an increased burden of cognitive dysfunction [15].

\section{Why PCKS9 Inhibitors Could Improve Cognition}

As with statins, the concern for short-term cognitive changes must be weighed against potential long-term protective effects. PCSK9 binds to the LDLR, inducing its degradation, and, therefore, increases serum LDL-C by reducing LDL clearance from the blood [16]. LDLR, which is also expressed in the brain, is responsible for clearing apolipoprotein $\mathrm{E}$ (apoE), a protein known to influence the accumulation of amyloid $\beta(A \beta)$ - the peptide responsible for Alzheimer's disease. LDLR is also upregulated by statins [17] and serves as one possible explanation for a decreased incidence of dementia in statin-treated patients [5].

Modulation of LDLR levels pharmacologically via PCSK9 inhibition can be considered as a potential strategy for dementia prevention. Indeed, knockout mice for the PCSK9 gene have been shown to have significantly lower 
apoE and plaque burden [18]. Controversy exists, however, as to whether circulating PCSK9 influences CNS expression of LDLR and, therefore, whether PCSK9 inhibitors could influence CNS lipid homeostasis [19, 20]. Rousselet et al. [21] showed in mice that PCSK9 inhibition did not interfere with brain development or recovery following an ischemic stroke, suggesting, at least via the interaction with LDLR, that these medications should not cause major cognitive harm. While this is reassuring, the translatability to humans and ability of injectable PCSK9 inhibitors to cross the blood-brain barrier in humans are important questions. PCSK9 inhibitors are antibodies which generally are unable to cross the blood-brain barrier without engineered "shuttle" molecules [22].

Alternatively, long-term lipid lowering through PCSK9 inhibition could lead to dementia prevention by improving arterial health. It is becoming increasingly recognized that traditional cardiovascular risk factors are not only risk factors for coronary and cerebrovascular events, but also for dementia [23, 24]. Triglyceride rich lipoproteins in particular are associated with small vessel cerebrovascular disease [25]. As with coronary artery disease, long-term lipid lowering may slow, halt, or even reverse the process of atherosclerosis in neurovascular network. Recognizing that the development of atherosclerosis and vascular dementia are not simply matters of cholesterol, it is conceivable that the arterial benefits of long-term lipid lowering by statins, PCSK9 inhibition, lifestyle changes, or other mechanisms could protect against vascular dementia.

\section{The Statin Experience}

Although concerns had surfaced in years prior, the concern that statin therapy may cause memory loss really came to the forefront of popular media and physician office visits in 2012 with the FDA label change. It was primarily based on reports from the Adverse Event Reporting System (AERS), a national case report database the FDA utilizes for drug safety surveillance. The FDA noted that symptoms were "ill-defined" and that the time to onset of the event was "highly variable, ranging from one day to years after statin exposure". A subsequent narrative review [27] of observational and randomized statin studies found no increased risk of cognitive decline and suggested that clinical practice should not change. Despite this, the public was not assured and scientific debate continued.

Much of the concern originated from the UCSD Statin Effects Study [2], which received particular attention in 2009. The study sought out patients who perceived side effects of statin therapy, and the majority (422/714; $59 \%)$ reported cognitive and/or memory problems. Of those reporting cognitive impairment, 171 (40\%) completed a "cognitive survey" indicating a median time of onset of 5 months, with recovery after statin cessation ranging from 1 day to multiple years. Of note, a very high proportion (70 \%) of these patients also reported muscle problems.

Although the UCSD study provides unique and important information, the ability for survey data to establish causality is limited, and there were serious concerns raised about both selection and reporting bias [27]. Regarding the latter bias, this group of investigators conducted a randomized trial on the effects of statins on cognition, with results available in 2004 [28], but have not published an article on the primary results, raising additional concerns about publication bias.

Since 2012, systematic reviews [5, 29-31] and narrative reviews [32-35] have repeatedly reported a lack of conclusive evidence that statins cause cognitive impairment. A Cochrane review in 2013 found that a mean reduction in LDL-C by $22 \%$ was not associated with worsening of cognitive function [36]. Most recently, Ott et al. [31] analyzed 27,643 participants from 14 randomized controlled trials (RCTs) finding that statin therapy was not associated with unfavorable effects on tests of cognition in those with or without impaired cognitive impairment at baseline. Richardson et al. [29] reached a similar conclusion citing moderate evidence that statin users did not experience an increase in mild cognitive impairment or change in global cognitive performance scores, executive function, declarative memory, processing speed, or visuoperception. Additionally, in our systematic review, analyzing 23,443 patients, we found that statin users in high-quality prospective cohort studies had a lower incidence of all-cause dementia [odds ratio (OR) 0.71, $95 \%$ confidence interval (CI) $0.61-0.82$; number needed to treat (NNT) 50, $95 \%$ CI 33-100] [5]. This protective effect has been noted by other investigators [37, 38].

In an important demonstration of the limitations of selfreported data, Richardson et al. [29] independently analyzed the FDA AERS database to calculate the reporting rates per prescription dispensed for statins, losartan, and clopidogrel. They found similar cognitive-related adverse reporting rates for all three drugs; however, clopidogrel and losartan have not received the same national attention or regulatory precautions as statins. Importantly, all three drug classes are often prescribed at the time of a cardiovascular event, which may impair cognition [39, 40].

\section{Limitations to Systematic Reviews}

While the systematic review evidence is reassuring, there are two notable limitations. The first limitation involves inadequate powering of subgroup analyses. Plausible arguments can be made that small subgroups of patients may 
be vulnerable to adverse effects and those effects are diluted out when analyzed with others. Such factors that may increase vulnerability are (1) use of a more lipophilic statin, increasing its ability to cross the blood-brain barrier; (2) intensity of the statin regimen, as determined by statin type and dose; and (3) underlying patient characteristics predisposing to adverse effects, such as pre-existing cognitive impairment, older age or genetic factors leading to higher circulating statin concentrations.

Wong et al. [37] noted no difference in the prevention of dementia with regard to statin lipophilicity $(0.94,95 \%$ CI $0.61-1.44$, vs. $1.07,0.70-1.63$ ), yet there is a paucity of head-to-head data between hydrophilic and lipophilic statins in the short-term cognitive domain, prohibiting an adequately powered analysis. The same is true in regard to statin intensity [29]. In terms of patients with cognitive impairment at baseline, four trials [41-43] examining the effect of statins on global cognitive function in those with Alzheimer's disease and one high-quality observational study [44] of patients with mild cognitive impairment have shown no worsening of function.

The second limitation is the quality of tools used to ascertain cognitive outcomes. While the limitations of subjectively reported adverse events are clear, the objective measures, such as the Mini-Mental State Examination (MMSE), employed by the vast majority of the aforementioned studies, may be too insensitive or unreliable for detecting the changes perceived by patients. This is especially true in cognitively normal patients, with test-retest reliability varying as much as $0.38-0.65$ for the MMSE [45]. In patients with Alzheimer's disease, subjective memory complaints have been shown to correlate with dementia-related anatomical changes, but predate decreases in MMSE scores, raising more concern about the sensitivity to detect mild impairment [46]. While objective testing targeting more specific domains of cognition such as declarative memory, attention, processing speed, visuoperception, and motor speed have also failed to show impairment in function with statin use, strength of evidence varies and the sensitivity for these tests for drug-related adverse effects is unknown [29].

\section{What We Know So Far About PCSK9 Inhibitors}

What appears to have prompted the FDA's directive was the Open-Label Study of Long-Term Evaluation Against LDL-C (OSLER) study, a safety, tolerability, and efficacy study from Amgen Inc., the developer of evolocumab [47]. The OSLER study enrolled patients from the initial 12-week phase II safety trials [47-50], with the goal of assessing safety at the end of 1 year. A cohort of 1104 hypercholesterolemic patients received $420 \mathrm{mg}$ of evolocumab every 4 weeks plus standard of care (SOC) or SOC alone. In the SOC group, $58 \%$ of patients were taking statins at baseline relative to $65 \%$ in the evolocumab + SOC cohort. Patients receiving evolocumab had a $52 \%$ reduction in LDL-C at 52 weeks compared with $2 \%$ in the SOC group. Overall, $81.4 \%$ of patients on evolocumab experienced an adverse event $(73.1 \%$ in SOC group), of which $3.7 \%$ required discontinuation of the treatment drug. The most common adverse events were nasopharyngitis (12\%), upper respiratory infections (8\%), arthralgias $(7 \%)$, muscle-related events $(9 \%)$ and injection-site reactions (5\%).

Cognitive side effects were uncommon in the OSLER study (Table 1$)$; three patients $(<1 \%)$ reported amnesia and five $(<1 \%)$ reported either memory or mental impairment. No events were reported in the SOC group. Of note, these patients were not blinded to treatment and no objective measures were performed. Interestingly, the adverse events were not related to the degree of LDL-C lowering such that clustering did not occur in the LDL-C $<25 \mathrm{mg} / \mathrm{dL}$ group $(n=98)$ relative to those in the $25-50 \mathrm{mg} / \mathrm{dL}(n=409)$ and $>50 \mathrm{mg} / \mathrm{dL}(n=323)$ groups.

More recent data from the OSLER program supports the earlier findings [52]. The OSLER investigators reported 11-month follow-up of two open-label, randomized trials involving 4465 patients who had participated in parent trials without having an adverse event leading to drug discontinuation. Neurocognitive events were reported in $0.9 \%$ of those allocated to evolocumab and $0.3 \%$ in the SOC group. This differential may be related, at least partly, to the unblinded study design and ascertainment bias

Table 1 Cognitive adverse events in the OSLER study [47]

\begin{tabular}{|c|c|c|c|c|}
\hline \multirow[t]{2}{*}{ Adverse events, $n(\%)$} & \multirow{2}{*}{$\begin{array}{l}\text { LDL-C }<25 \mathrm{mg} / \mathrm{dL}^{\mathrm{a}} \\
\text { Evolocumab }+ \text { SOC } \\
(n=98)\end{array}$} & \multirow{2}{*}{$\begin{array}{l}\text { LDL-C }<50 \mathrm{mg} / \mathrm{dL}^{\mathrm{a}} \\
\text { Evolocumab }+ \text { SOC } \\
(n=409)\end{array}$} & \multicolumn{2}{|c|}{ LDL-C $\geq 50 \mathrm{mg} / \mathrm{dL}$} \\
\hline & & & $\begin{array}{l}\text { SOC } \\
(n=359)\end{array}$ & $\begin{array}{l}\text { Evolocumab + SOC } \\
(n=323)\end{array}$ \\
\hline Amnesia & $1(1.0)$ & $1(0.2)$ & $0(0.0)$ & $1(0.3)$ \\
\hline Memory or mental impairment & $0(0.0)$ & $4(1.0)$ & $0(0.0)$ & $1(0.3)$ \\
\hline
\end{tabular}

$L D L-C$ low-density lipoprotein cholesterol, $S O C$ standard of care

${ }^{\text {a }}$ No patients in the SOC group had LDL-C $<25 \mathrm{mg} / \mathrm{dL}$ and two patients had LDL-C $<50 \mathrm{mg} / \mathrm{dL}$ 
because participants on PCSK9 inhibitors were required to attend more in-person visits. Moreover, very low LDL-C levels of $<25 \mathrm{mg} / \mathrm{dL}$ raise a new safety concern; however, there was not a significant association of neurocognitive reports with on-treatment LDL-C levels.

The ODYSSEY LONG TERM trial, using alirocumab, showed a similar numerically higher proportion of neurocognitive adverse events in the active treatment group of 1.2 versus $0.5 \%$ in the placebo group [52]. The reported adverse events were mostly related to memory. Although the difference between the groups did not reach formal statistical significance $(p=0.17)$, it is notably similar to the signal in OSLER. In contrast to OSLER, ODYSSEY LONG TERM participants were blinded and follow-up was somewhat longer (78 weeks).

It is worth mentioning the other side of the coin: cardiovascular events. Though neither OSLER nor ODYSSEY LONG TERM was designed primarily as a hard outcome trial, they offer early looks at the potential for PCSK9 inhibitors to confer protection from cardiovascular events. A pre-specified exploratory analysis in OSLER showed a cardiovascular event rate of $2.18 \%$ in the SOC group compared with $0.95 \%$ in the evolocumab group [hazard ratio (HR) $0.47,95 \%$ CI $0.28-0.78, p=0.003]$. In ODYSSEY LONG TERM, there was a similar approximate $50 \%$ relative risk reduction in cardiovascular events.

\section{Ongoing PCSK9 Studies}

There are four ongoing phase III trials (Fig. 1) evaluating major adverse cardiac events, ODYSSEY OUTCOMES [53], FOURIER [54], SPIRE-1 [55], and SPIRE-2 [56], with results expected in 2017 and 2018. All four companies-Pfizer (bococizumab), Amgen (evolocumab), and Sanofi/Regeneron (alirocumab)_released press releases stating they are in communication with the FDA regarding potential neurocognitive adverse events and have seen no safety signals to date in these late-stage trials [1, 57]. While it remains mostly unclear whether these events will be subjectively reported or objectively determined (via neurocognitive testing), all four companies plan to monitor for such side effects and report results from their late-stage trials.

We are aware of one dedicated neurocognitive substudy sponsored by Amgen [58]: evaluating PCSK9 Binding antiBody Influence oN coGnitive HeAlth in High cardiovascUlar Risk Subjects (EBBINGHAUS) (NCT02207634). FOURIER trial participants free of current or past dementia or mild cognitive impairment are eligible, with an expected enrollment of 4000 participants. The primary outcome is mean change from baseline over time in the Spatial Working Memory (SWM) index of executive function.
Secondary outcomes include mean changes from baseline over time in the SWM between-errors score and adjusted Paired Associated Learning total errors, and median 5-Choice Reaction Time. The study began in August 2014, and estimated completion is in September 2017. It will provide important data as a specific assessment of neurocognitive function with longer-term follow-up.

\section{Surveillance for Cognitive Dysfunction with PCSK9 Inhibitors: A Case for $N$-of-1 Trials}

As with the statin experience, even if ongoing PCSK9 trials do not show any overall risk of adverse neurocognitive effects, it is unlikely that this will provide complete reassurance or settle debate. There could still be hidden vulnerable subgroups, and even if proportionally small, the sheer market potential for these drugs means the absolute size of such a group could be considerable. Statins as a class were prescribed 255.4 million times in 2010 [59] and, with an estimated 10-20\% of patients "intolerant to statins", and many more high-risk patients not attaining an optimal LDL-C on statin monotherapy [60], PCSK9 inhibitors have the potential to be used in millions of patients around the globe. PCSK9 inhibitors can also lower LDL-C to a greater degree than is seen with even the strongest statin therapy, which raises concern about a dose-response effect.

The FDA has issued an important directive about cognitive safety monitoring; however, what remains unclear is whether traditional clinical trials are the best approach. Randomized clinical trials are the sine que non of medical research, but are best utilized to determine group averaged effects on common outcomes, such as major adverse cardiac events, with objective means of measure. What seems desirable is a study design that can incorporate the randomized, blinded rigor of randomized trials with the targeted patient-centeredness of case reports and case series. Such a design may offer scientific purists the desired level of evidence while offering other experts the desired attention to patients who deviate from the group average.

To bridge the divide between both sides of the debate, we suggest $n$-of- 1 trials. An $n$-of- 1 trial is a single patient crossover trial in which a patient is randomized to active drug or placebo in blinded fashion for specified periods of time. Multiple crossovers may be desired. $N$-of- 1 trials may be better suited to establish causality, be less costly, and be a more efficient means of pursuing adverse cognitive events in patients exposed to PCSK9 inhibitors and statins. Following the completion of ongoing PCSK9 trials, those participants who reported an adverse neurocognitive event could be offered the opportunity to participate in an $n$-of- 1 trial. Moreover, if PCSK9 inhibitors are approved, then the 


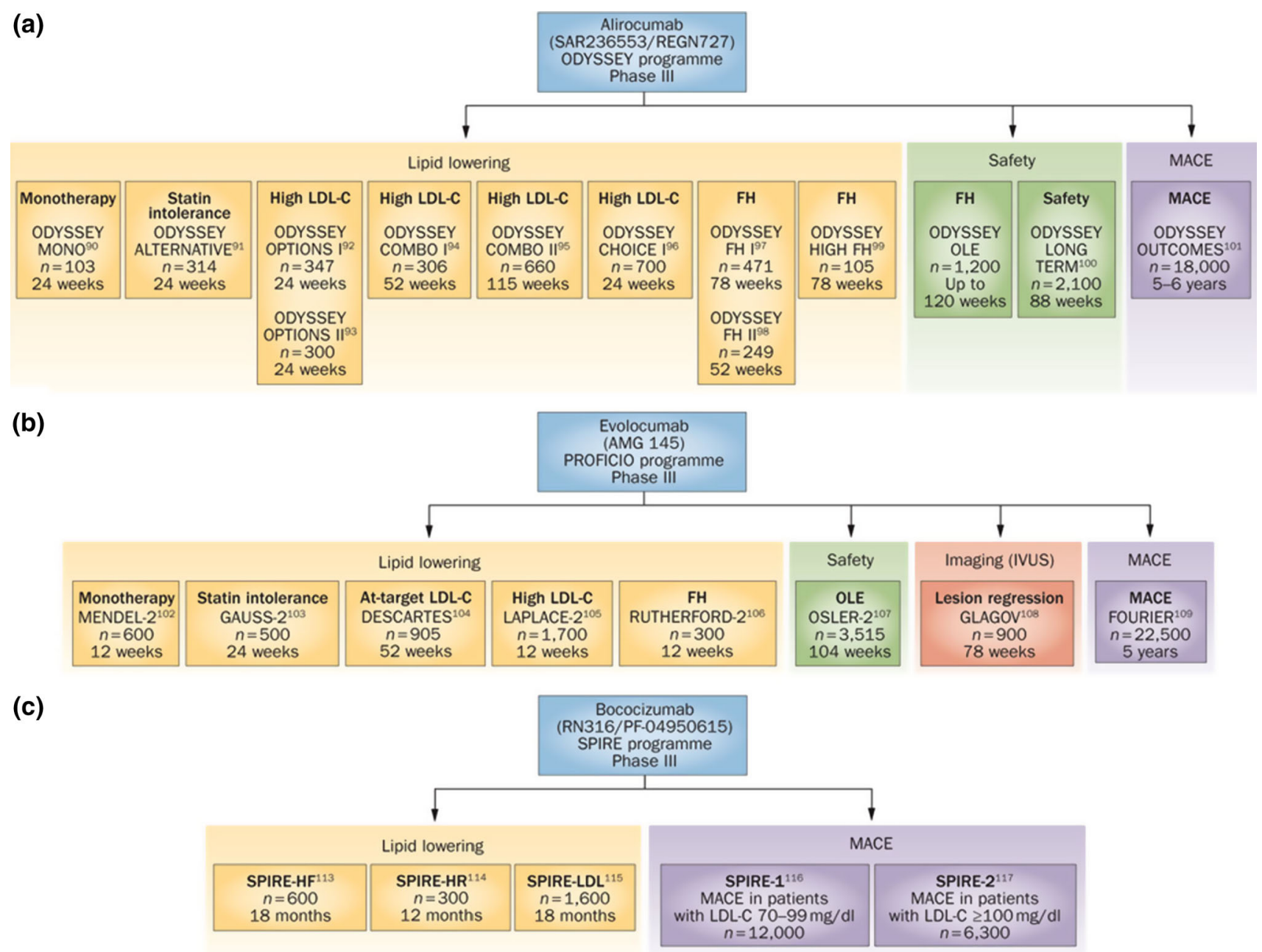

Fig. 1 Ongoing phase III clinical trials of PCSK9 inhibitors. Phase III trials designed to evaluate the long-term safety and tolerability of a alirocumab, $\mathbf{b}$ evolocumab, and $\mathbf{c}$ bococizumab, and their efficacy in reducing the rate of cardiovascular events in various populations of patients. FH familial hypercholesterolemia, IVUS intravascular ultrasonography, $L D L-C$ low-density lipoprotein cholesterol, $M A C E$ major adverse cardiac events, $O L E$ open-label extension, PCSK9 proprotein convertase subtilisin-kexin type 9. Reproduced from Dadu et al. [62] with permission from Nature Reviews Cardiology

FDA could develop a program to select post-approval surveillance cases from the AERS database and offer those patients the opportunity to undergo testing using an $n$-of- 1 design. Individuals would receive objective neurocognitive testing in addition to keeping a symptom diary during blinded, random allocations to PCSK9 inhibition and placebo, with a washout period between crossovers. Through these means, causality could be more definitively assessed in a patient-centered approach. This approach would also be free of ascertainment bias introduced to PCSK9 trials by more closely monitoring patients on active study drug.

Although the $n$-of-1 approach has not been applied in statin-related cognitive dysfunction, to the best of our knowledge, it has shown usefulness for statin-related myalgias [61]. Joy et al. [61] enrolled eight patients with prior statin-related myalgia from a tertiary care lipid clinic in $n$-of-1 trials with three double-blind, crossovers separated by 3-week washouts. The primary outcome was the visual analog scale myalgia score, which patients reported weekly. Scores did not differ in any of the $n$-of- 1 trials, and when patients were presented with these results, five patients chose to resume statin treatment. Therefore, this limited experience with the $n$-of- 1 trial in assessing statin safety may serve as a useful model that can be considered for efficient, rigorous, and patient-centered safety assessments of PCSK9 inhibitors.

\section{Conclusion}

In conclusion, neurocognitive impairment is theoretically possible with any lipid-lowering therapy. The statin experience indicates that apparently discordant reports of 
neurocognitive adverse effects may be related to differences in research methodologies with inherent tradeoffs in risk of bias and patient-centeredness. On both theoretical and empirical grounds, concern for adverse neurocognitive effects currently extends to PCSK9 inhibitors. Available evidence from PCSK9 trials suggests an uncommon effect, but is inconclusive; ongoing trials providing more data, longer-term data, and objective testing will be informative. Still, the statin experience indicates that traditional RCT evidence is insufficient to settle debate given its inherent focus on group averages. As a solution to this problem, we propose that $n$-of- 1 trials warrant close consideration in moving forward with PCSK9 inhibitor safety evaluation. Having safety data of the highest possible quality is critical to best inform the shared decision-making process that occurs when a patient and his or her clinician weigh potential benefits and risks of any lipid-lowering therapy.

Compliance with Ethical Standards Funding No sources of funding were used to assist in the preparation of this study.

Conflict of interest Kristopher J. Swiger has no conflict of interests that are directly relevant to the content of this study. Seth S. Martin is supported by the Pollin Cardiovascular Prevention Fellowship, MarieJosée and Henry R. Kravis endowed fellowship, and a National Institutes of Health training Grant (T32HL07024). He is also listed as a co-inventor on a pending patent filed by Johns Hopkins University for a method of LDL-C estimation.

\section{References}

1. FDA advises of adverse effects from new cholesterol drugsWSJ [Internet]. [cited $2015 \mathrm{Feb} 20$ ]. Available from: http://www. wsj.com/articles/SB100014240527023047328045794256124879 04436. Accessed 20 Feb 2015.

2. Evans MA, Golomb BA. Statin-associated adverse cognitive effects: survey results from 171 patients. Pharmacotherapy. 2009;29:800-11.

3. FDA drug safety communication [Internet]. [cited 2012 Aug 7]. Available from: http://www.fda.gov/Drugs/DrugSafety/ucm2931 01.htm. Accessed 7 Aug 2012.

4. Stone NJ, Robinson J, Lichtenstein AH, Merz CNB, Blum CB, Eckel RH, et al. 2013. ACC/AHA Guideline on the treatment of blood cholesterol to reduce atherosclerotic cardiovascular risk in adults: a report of the American College of Cardiology/American Heart Association Task Force on Practice Guidelines. Circulation. 2014;129(25 Suppl 2):S1-45.

5. Swiger KJ, Manalac RJ, Blumenthal RS, Blaha MJ, Martin SS. Statins and cognition: a systematic review and meta-analysis of short- and long-term cognitive effects. Mayo Clin Proc Elsevier Inc. 2013;88(11):1213-21.

6. Björkhem I, Meaney S. Brain cholesterol: long secret life behind a barrier. Arterioscler Thromb Vasc Biol. 2004;24:806-15.

7. Joseph JA, Denisova N, Villalobos-Molina R, Erat S, Strain J. Oxidative stress and age-related neuronal deficits. Mol Chem Neuropathol. 1996;28:35-40.

8. Vecka M, Tvrzická E, Stanková B, Novák F, Nováková O, Zák A. Hypolipidemic drugs can change the composition of rat brain lipids. Tohoku J Exp Med. 2004;204:299-308.
9. Wahler JB, Swain MG, Carson R, Bergasa NV, Jones EA. Bloodbrain barrier permeability is markedly decreased in cholestasis in the rat. Hepatology. 1993;17:1103-8.

10. Blom DJ, Hala T, Bolognese M, Lillestol MJ, Toth PD, Burgess L, et al. A 52-week placebo-controlled trial of evolocumab in hyperlipidemia. N Engl J Med. 2014;370:1809-19.

11. Robinson JG, Nedergaard BS, Rogers WJ, Fialkow J, Neutel JM, Ramstad D, et al. Effect of evolocumab or ezetimibe added to moderate- or high-intensity statin therapy on LDL-C lowering in patients with hypercholesterolemia: the LAPLACE-2 randomized clinical trial. JAMA. 2014;311:1870-82.

12. Stroes E, Colquhoun D, Sullivan D, Civeira F, Rosenson RS, Watts GF, et al. Anti-PCSK9 antibody effectively lowers cholesterol in patients with statin intolerance: the GAUSS-2 randomized, placebo-controlled phase 3 clinical trial of evolocumab. J Am Coll Cardiol. 2014;63:2541-8.

13. Kuklina EV, Yoon PW, Keenan NL. Trends in high levels of lowdensity lipoprotein cholesterol in the United States, 1999-2006. JAMA. 2009;302:2104-10.

14. Martin SS, Blumenthal RS, Miller M. LDL cholesterol: the lower the better. Med Clin North Am (Elsevier Inc). 2012;96:13-26.

15. Postmus I, Trompet S, de Craen AJM, Buckley BM, Ford I, Stott DJ, et al. PCSK9 SNP rs11591147 is associated with low cholesterol levels but not with cognitive performance or noncardiovascular clinical events in an elderly population. J Lipid Res. 2013;54:561-6.

16. Lagace TA. PCSK9 and LDLR degradation: regulatory mechanisms in circulation and in cells. Curr Opin Lipidol. 2014;25:387-93.

17. Goldstein JL, Brown MS. Regulation of the mevalonate pathway. Nature. 1990;343:425-30.

18. Bagchi D. Regulation of amyloid plaque deposition by PCSK9 in a mouse model of Alzheimer's disease. Undergrad. ThesesRestricted 2011.

19. Liu M, Wu G, Baysarowich J, Kavana M, Addona GH, Bierilo $\mathrm{KK}$, et al. PCSK9 is not involved in the degradation of LDL receptors and BACE1 in the adult mouse brain. J Lipid Res. 2010;51:2611-8.

20. Poirier S, Mayer G, Benjannet S, Bergeron E, Marcinkiewicz J, Nassoury N, et al. The proprotein convertase PCSK9 induces the degradation of low density lipoprotein receptor (LDLR) and its closest family members VLDLR and ApoER2. J Biol Chem. 2008;283:2363-72.

21. Rousselet E, Marcinkiewicz J, Kriz J, Zhou A, Hatten ME, Prat A, et al. PCSK9 reduces the protein levels of the LDL receptor in mouse brain during development and after ischemic stroke. J Lipid Res. 2011;52:1383-91.

22. Niewoehner J, Bohrmann B, Collin L, Urich E, Sade H, Maier P, Rueger P, Stracke J, Lau W, Tissot A, Loetscher H, Ghosh A, Freskgard $\mathrm{P}$. Increased brain penetration and potency of a therapeutic antibody using a monovalent molecular shuttle. Neuron. 2014;81:49-60.

23. Hasnain M, Vieweg WVR. Possible role of vascular risk factors in Alzheimer's disease and vascular dementia. Curr Pharm Des. 2014;20:6007-13.

24. Douiri A, McKevitt C, Emmett ES, Rudd AG, Wolfe CDA. Long-term effects of secondary prevention on cognitive function in stroke patients. Circulation. 2013;128:1341-8.

25. Schilling S, Tzourio C, Dufouil C, Zhu Y, Berr C, Alpérovitch A, et al. Plasma lipids and cerebral small vessel disease. Neurology. 2014;83:1844-52.

26. Jukema JW, Cannon CP, de Craen AJM, Westendorp RGJ, Trompet $\mathrm{S}$. The controversies of statin therapy: weighing the evidence. J Am Coll Cardiol (American College of Cardiology Foundation). 2012;60:875-81 
27. Martin S, Blumenthal R, Blaha M. Let's not be so quick to stop statins [Internet]. Healio. 2012. Available from: http://www. healio.com/cardiology/vascular-medicine/news/print/cardiologytoday/\%7B3b6a1c09-bb29-4078-a59e-5a7d8f492922\%7D/letsnot-be-so-quick-to-stop-statins. Accessed 20 Feb 2015.

28. US National Library of Medicine [Internet]. ClinicalTrials.gov. Available from: https://clinicaltrials.gov/ct2/show/NCT0033098 0?term=NCT00330980\&rank=1. Accessed 20 Feb 2015.

29. Richardson K, Schoen M, French B, Umscheid CA, Mitchell MD, Arnold SE, et al. Statins and cognitive function: a systematic review. Ann Intern Med. 2013;159:688-97.

30. McGuinness B, O'Hare J, Craig D, Bullock R, Malouf R, Passmore P. Cochrane review on "Statins for the treatment of dementia". Int J Geriatr Psychiatry. 2013;28:119-26.

31. Ott BR, Daiello LA, Dahabreh IJ, Springate BA, Bixby K, Murali $\mathrm{M}$, et al. Do statins impair cognition? A systematic review and meta-analysis of randomized controlled trials. J Gen Intern Med. 2015;30:348-58.

32. Kelley BJ, Glasser S. Cognitive effects of statin medications. CNS Drugs. 2014;28:411-9.

33. Tuccori M, Montagnani S, Mantarro S, Capogrosso-Sansone A, Ruggiero E, Saporiti A, et al. Neuropsychiatric adverse events associated with statins: epidemiology, pathophysiology, prevention and management. CNS Drugs. 2014;28:249-72.

34. Strandberg T, Strandberg A. [Statin therapy and cognition]. Duodecim. 2013;129:713-8.

35. Desai CS, Martin SS, Blumenthal RS. Non-cardiovascular effects associated with statins. BMJ. 2014;349:g3743.

36. McGuinness B, Craig D, Bullock R, Malouf R, Passmore P. Statins for the treatment of dementia. Cochrane Database Syst Rev. 2014;7:CD007514.

37. Wong WB, Lin VW, Boudreau D, Devine EB. Statins in the prevention of dementia and Alzheimer's disease: a meta-analysis of observational studies and an assessment of confounding. Pharmacoepidemiol Drug Saf. 2013;22:345-58.

38. Song Y, Nie $\mathrm{H}, \mathrm{Xu} \mathrm{Y,} \mathrm{Zhang} \mathrm{L,} \mathrm{Wu} \mathrm{Y.} \mathrm{Association} \mathrm{of} \mathrm{statin} \mathrm{use}$ with risk of dementia: a meta-analysis of prospective cohort studies. Geriatr Gerontol Int. 2013;13(4):817-24.

39. Gharacholou SM, Reid KJ, Arnold SV, Spertus J, Rich MW, Pellikka PA, et al. Cognitive impairment and outcomes in older adult survivors of acute myocardial infarction: findings from the translational research investigating underlying disparities in acute myocardial infarction patients' health status registry. Am Heart J (Elsevier). 2011;162:860-869.e1.

40. Breteler MM, Claus JJ, Grobbee DE, Hofman A. Cardiovascular disease and distribution of cognitive function in elderly people: the Rotterdam Study. BMJ. 1994;308:1604-8.

41. Feldman HH, Doody RS, Kivipelto M, Sparks DL, Waters DD, Jones RW, et al. Randomized controlled trial of atorvastatin in mild to moderate Alzheimer disease: LEADe. Neurology. 2010;74:956-64.

42. Sano M, Bell KL, Galasko D, Galvin JE, Thomas RG, van Dyck CH, et al. A randomized, double-blind, placebo-controlled trial of simvastatin to treat Alzheimer disease. Neurology. 2011;77:556-63.

43. Simons M, Schwärzler F, Lütjohann D, von Bergmann K, Beyreuther K, Dichgans J, et al. Treatment with simvastatin in normocholesterolemic patients with Alzheimer's disease: a 26-week randomized, placebo-controlled, double-blind trial. Ann Neurol. 2002;52:346-50.

44. Sparks DL, Kryscio RJ, Connor DJ, Sabbagh MN, Sparks LM, Lin Y, et al. Cholesterol and cognitive performance in normal controls and the influence of elective statin use after conversion to mild cognitive impairment: results in a clinical trial cohort. Neurodegener Dis. 2010;7:183-6.

45. Hensel A, Angermeyer MC, Riedel-Heller SG. Measuring cognitive change in older adults: reliable change indices for the Mini-
Mental State Examination. J Neurol Neurosurg Psychiatry. 2007;78:1298-303.

46. Jessen F, Feyen L, Freymann K, Tepest R, Maier W, Heun R, et al. Volume reduction of the entorhinal cortex in subjective memory impairment. Neurobiol Aging. 2006;27:1751-6.

47. Koren MJ, Giugliano RP, Raal FJ, Sullivan D, Bolognese M, Langslet G, et al. Efficacy and safety of longer-term administration of evolocumab (AMG 145) in patients with hypercholesterolemia: 52-week results from the Open-Label Study of LongTerm Evaluation Against LDL-C (OSLER) randomized trial. Circulation. 2014;129:234-43.

48. Sullivan D, Olsson AG, Scott R, Kim JB, Xue A, Gebski V, et al. Effect of a monoclonal antibody to PCSK9 on low-density lipoprotein cholesterol levels in statin-intolerant patients: the GAUSS randomized trial. JAMA. 2012;308:2497-506.

49. Giugliano RP, Desai NR, Kohli P, Rogers WJ, Somaratne R, Huang F, et al. Efficacy, safety, and tolerability of a monoclonal antibody to proprotein convertase subtilisin/kexin type 9 in combination with a statin in patients with hypercholesterolaemia (LAPLACE-TIMI 57): a randomised, placebo-controlled, doseranging, phase 2 study. Lancet. 2012;380:2007-17.

50. Raal F, Scott R, Somaratne R, Bridges I, Li G, Wasserman SM, et al. Low-density lipoprotein cholesterol-lowering effects of AMG 145, a monoclonal antibody to proprotein convertase subtilisin/kexin type 9 serine protease in patients with heterozygous familial hypercholesterolemia: the Reduction of LDL-C with PCSK9 Inhibition in Heterozygous Familial Hypercholesterolemia Disorder (RUTHERFORD) randomized trial. Circulation. 2012;126:2408-17.

51. Sabatine MS, Giugliano RP, Wiviott SD, Raal FJ, Blom DJ, Robinson J, et al. Efficacy and safety of evolocumab in reducing lipids and cardiovascular events. N Engl J Med. 2015;372(16): 1500-9.

52. Robinson JG, Farnier M, Krempf M, Bergeron J, Luc G, Averna $\mathrm{M}$, et al. Efficacy and safety of alirocumab in reducing lipids and cardiovascular events. N Engl J Med. 2015;372(16):1489-99.

53. US National Library of Medicine [Internet]. ClinicalTrials.gov. Available from: https://clinicaltrials.gov/ct2/show/NCT01663402. Accessed 20 Feb 2015.

54. US National Library of Medicine [Internet]. ClinicalTrials.gov. Available from: https://clinicaltrials.gov/ct2/show/NCT01764633. Accessed 20 Feb 2015.

55. US National Library of Medicine [Internet]. ClinicalTrials.gov. Available from: https://clinicaltrials.gov/ct2/show/NCT01975376. Accessed 20 Feb 2015.

56. US National Library of Medicine [Internet]. ClinicalTrials.gov. Available from: https://clinicaltrials.gov/ct2/show/NCT01975389. Accessed 20 Feb 2015.

57. Ballantyne CM, Neutel J, Cropp A, Duggan W, Wang EQ, Plowchalk D, et al. Results of bococizumab, a monoclonal antibody against proprotein convertase subtilisin/kexin type 9 , from a randomized, placebo-controlled, dose-ranging study in statintreated subjects with hypercholesterolemia. Am J Cardiol. 2015;115:1212-21.

58. US National Library of Medicine [Internet]. ClinicalTrials.gov. Available from: https://clinicaltrials.gov/ct2/show/NCT02207634. Accessed 20 Feb 2015.

59. The use of medicines in the United States: review of 2010. 2011.

60. Fitchett DH, Hegele RA, Verma S. Statin intolerance. Circulation. 2015;131:e389-91.

61. Joy TR, Monjed A, Zou GY, Hegele RA, McDonald CG, Mahon JL. N-of-1 (single-patient) trials for statin-related myalgia. Ann Intern Med. 2014;160:301-10.

62. Dadu R, Ballantyne C. Lipid lowering with PCSK9 inhibitors. Nat Rev Cardiol (Nature Publishing Group). 2014;11:563-75. 